Article

\title{
Influence of Pressure on the Mechanical and Electronic Properties of Wurtzite and Zinc-Blende GaN Crystals
}

\author{
Hongbo Qin ${ }^{1, * \mathbb{D}}$, Tianfeng Kuang ${ }^{1}$, Xinghe Luan ${ }^{1}$, Wangyun $\mathrm{Li}^{1}{ }^{1}$, Jing Xiao ${ }^{1}$, \\ Ping Zhang 1,2,*(D), Daoguo Yang ${ }^{1,3}$ and Guoqi Zhang 3 \\ 1 School of Mechanical and Electronic Engineering, Guilin University of Electronic Technology, Guilin 541004, \\ China; 1701302022@mails.guet.edu.cn (T.K.); 1601201040@mails.guet.edu.cn (X.L.); \\ li.wangyun@guet.edu.cn (W.L.); xiaojing@guet.edu.cn (J.X.); daoguo_yang@163.com (D.Y.) \\ 2 MIIT Key Laboratory of Thermal Control of Electronic Equipment, School of Energy and Power Engineering, \\ Nanjing University of Science \& Technology, Nanjing 210094, China \\ 3 EEMCS Faculty, Delft University of Technology, 2628 Delft, The Netherlands; g.q.zhang@tudelft.nl \\ * Correspondence: qinhb@guet.edu.cn (H.Q.); zp3631@gmail.com (P.Z.)
}

Received: 19 October 2018; Accepted: 8 November 2018; Published: 14 November 2018

\begin{abstract}
The mechanical and electronic properties of two GaN crystals, wurtzite and zinc-blende $\mathrm{GaN}$, under various hydrostatic pressures were investigated using first principles calculations. The results show that the lattice constants of the two GaN crystals calculated in this study are close to previous experimental results, and the two GaN crystals are stable under hydrostatic pressures up to $40 \mathrm{GPa}$. The pressure presents extremely similar trend effect on the volumes of unit cells and average Ga-N bond lengths of the two GaN crystals. The bulk modulus increases while the shear modulus decreases with the increase in pressure, resulting in the significant increase of the ratios of bulk moduli to shear moduli for the two GaN polycrystals. Different with the monotonic changes of bulk and shear moduli, the elastic moduli of the two GaN polycrystals may increase at first and then decrease with increasing pressure. The two GaN crystals are brittle materials at zero pressure, while they may exhibit ductile behaviour under high pressures. Moreover, the increase in pressure raises the elastic anisotropy of GaN crystals, and the anisotropy factors of the two GaN single crystals are quite different. Different with the obvious directional dependences of elastic modulus, shear modulus and Poisson's ratio of the two GaN single crystals, there is no anisotropy for bulk modulus, especially for that of zinc-blende GaN. Furthermore, the band gaps of GaN crystals increase with increasing pressure, and zinc-blende $\mathrm{GaN}$ has a larger pressure coefficient. To further understand the pressure effect on the band gap, the band structure and density of states (DOSs) of GaN crystals were also analysed in this study.
\end{abstract}

Keywords: GaN; pressure; first principle; mechanical property; electronic property

\section{Introduction}

Because of superior performances, such as wide direct band gap, excellent luminous efficiency, corrosion resistance, low dielectric constant, high temperature resistance, electron mobility and outstanding mechanical strength [1-5], GaN is known as one of the third-generation semiconductor materials and can be applied potentially in LEDs, lasers, sensors, high power, spintronic devices, etc. [6,7]. Usually, GaN has a hexagonal wurtzite structure. It has been reported that, in a revised molecular-beam epitaxy system, cubic zinc-blende GaN can be obtained on $\beta$-SiC (100) substrates [8]. Compared with wurtzite GaN, zinc-blende $\mathrm{GaN}$ is supposed to be more flexible for molecular designs [9,10]. There have been numerous works about the effect of surface adsorption [11-14], 
defects [15-17] and doping [18-21] on the electronic and optoelectronic performances of wurtzite GaN, while less research has been done about that of zinc-blende GaN. Thus far, because of deficiencies of experimental equipment and measurement methods, in-depth investigations on the mechanical and electronic performances of the two GaN crystals are still limited.

First principles calculations about the mechanical and physical performances of materials have been successfully carried out and the accuracy of their results has been verified [22-24]. Wang and Ye [25] investigated the lattice parameters, bulk moduli and energy band structures of twelve III-V wurtzite and zinc-blende semiconductors under pressure via the first principle calculation, and the pressure dependences of band gaps of different semiconductors were presented. However, relevant information about the GaN in Wang and Ye's study is still very limited. In order to further understand the mechanical and physical performances of the two GaN crystals, to help establish finite element models and to evaluate the reliability of components and devices, first-principles calculations using density functional theory (DFT) have been applied in this study to explore the mechanical and electronic properties of wurtzite and zinc-blende GaN crystals under different hydrostatic pressures.

\section{Computational Methods}

The CASTEP programme was applied to perform DFT calculations [26]. The generalised gradient approximation (GGA) of the revised Perdew-Burke-Ernzerhof (PBE) formalism $[27,28]$ and the local density approximation (LDA) proposed by Ceperley and Alder and parameterized by Perdew and Zunger (CA-PZ) $[29,30]$ were used to calculate the exchange-correlation potential. Meanwhile, to optimise the GaN crystals structures, Vanderbilt ultra-soft pseudopotentials [31] and the BFGS algorithm [21] were employed. The self-consistent field tolerance and plane wave basis cut-off energy were set at $5.0 \times 10^{-7} \mathrm{eV}$ atom ${ }^{-1}$ and $520 \mathrm{eV}$, respectively. To guarantee the variation of total energy was smaller than $1 \mathrm{meV}$ atom $^{-1}$ [22,32], a $12 \times 12 \times 12$ Monkhorst-Pack grid [33] was chosen in the calculation for the Brillouin-zone integration. The convergence tolerance of the energy was $5.0 \times 10^{-6} \mathrm{eV}$, and those for maximum force, maximum displacement and maximum stress were set at $0.01 \mathrm{eV} / \AA, 0.0005 \AA$ and $20 \mathrm{MPa}$, respectively. For providing a good representation of the electron density, $25 \times 25 \times 25$ mesh was selected for the fast Fourier transform grid parameters. As for relativistic treatment, the Koeling-Harmon method was employed [34]. At the same time, density mixing was used to specify the electronic minimization method for the self-consistent field calculation [35]. By using linear fitting stress values at four small strains \pm 0.001 and \pm 0.003 , the elastic stiffness matrix $C_{i j}$ could be calculated according to nine deformation conditions [36]. Then, as the inverse matrix of $C_{i j}$, the compliance matrix $S_{i j}$ was obtained. The structural, elastic and electronic properties of GaN crystals were explored under external hydrostatic pressures ranging from 0 to $40 \mathrm{GPa}$.

\section{Results and Discussion}

\subsection{Structural Properties}

The wurtzite GaN crystal has a hexagonal structure and its space group is P63mc [37], see Figure 1a. Zinc-blende GaN crystallises in a cubic structure, and has the space group F-43m [37], as displayed in Figure $1 \mathrm{~b}$. After geometry optimization, the lattice parameters were calculated; results are given in Table 1. Clearly, the calculation results in this study fit well with the experimental data and calculation results listed in previous studies, proving the validity of the calculations in the present study. Generally, GGA overestimates the lattice parameters while LDA underestimates them, and the lattice parameters obtained from LDA are much closer to experimental data. Our previous work revealed that, compared to GGA, LDA is more accurate in predicting elastic properties [38]. Therefore, LDA was applied in the following study.

The ratio of $V$ to $V_{0}$ is applied to evaluate the relative change in unit cell volume under different pressures, where $V$ and $V_{0}$ are volumes of the unit cell at a specified pressure and zero pressure, respectively. Pressure dependences of $V / V_{0}$ of the two GaN crystals are shown in Figure 2a. 
Obviously, $V / V_{0}$ value decreased substantially with the increasing hydrostatic pressure, and the pressure presented an extremely similar trend effect on $V$ of the two GaN crystals. This is due to the fact that the average bond lengths of the two GaN crystals are also very close to each other under the same pressure, see Figure $2 b$.

(a)

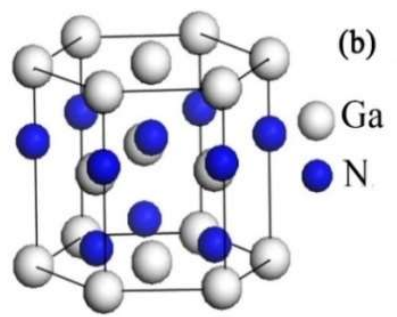

(b)

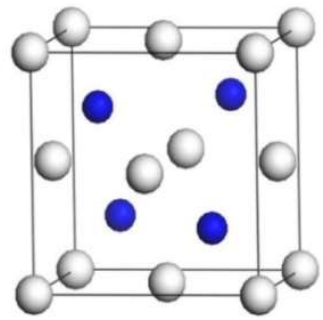

(c)

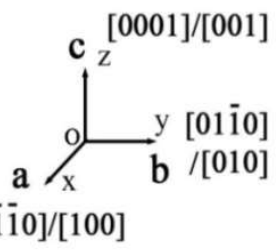

Figure 1. Structures of GaN crystals: (a) wurtzite GaN; (b) zinc-blende GaN; and (c) crystallographic axes and directions.
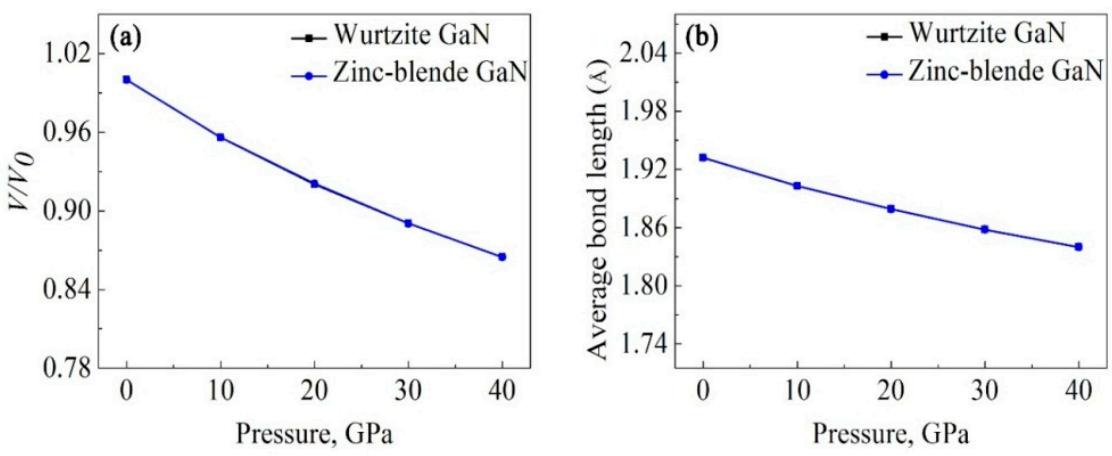

Figure 2. Calculated pressure dependences of $V / V_{0}(\mathbf{a})$ and average Ga-N bond lengths $(\mathbf{b})$.

Table 1. Lattice parameters at zero pressure.

\begin{tabular}{|c|c|c|c|c|c|c|}
\hline \multirow{2}{*}{ Phase } & \multirow{2}{*}{ Approach } & \multicolumn{5}{|c|}{ Lattice Parameters } \\
\hline & & $a_{0}(\AA)$ & $b_{0}(\AA)$ & $c_{0}(\AA)$ & $c_{0} / a_{0}$ & $V_{0}$ \\
\hline \multirow{8}{*}{$\begin{array}{l}\text { Wurtzite GaN } \\
\qquad(P 63 m c)\end{array}$} & GGA (present) & 3.246 & 3.246 & 5.280 & 1.627 & 48.185 \\
\hline & LDA (present) & 3.159 & 3.159 & 5.145 & 1.629 & 44.477 \\
\hline & GGA [39] & 3.199 & 3.199 & 5.227 & 1.634 & 46.306 \\
\hline & LDA [39] & 3.131 & 3.131 & 5.104 & 1.630 & 43.331 \\
\hline & GGA [40] & 3.245 & 3.245 & 5.296 & 1.632 & 48.296 \\
\hline & LDA [40] & 3.193 & 3.193 & 5.281 & 1.634 & 46.628 \\
\hline & GGA [41] & 3.197 & 3.197 & 5.210 & 1.630 & 46.118 \\
\hline & Expt. [42] & 3.189 & 3.189 & 5.185 & 1.626 & 45.671 \\
\hline \multirow{6}{*}{$\begin{array}{c}\text { Zinc-blendeGaN } \\
(F-43 m)\end{array}$} & GGA (present) & 4.582 & 4.582 & 4.582 & 1.000 & 96.217 \\
\hline & LDA (present) & 4.461 & 4.461 & 4.461 & 1.000 & 88.786 \\
\hline & GGA [43] & 4.550 & 4.550 & 4.550 & 1.000 & 94.196 \\
\hline & LDA [40] & 4.518 & 4.518 & 4.518 & 1.000 & 92.223 \\
\hline & LDA [39] & 4.446 & 4.446 & 4.446 & 1.000 & 87.884 \\
\hline & Expt. [9] & 4.490 & 4.490 & 4.490 & 1.000 & 90.519 \\
\hline
\end{tabular}

\subsection{Elastic Constants}

Elastic constants, $C_{i j}$, can quantify the response of deformation under a force or stress load. In the stiffness matrix of the hexagonal structure-like wurtzite $\mathrm{GaN}$ crystal, there are five independent elastic constants, i.e., $C_{11}, C_{12}, C_{13}, C_{33}$ and $C_{44}$, which should conform the stability criteria [44]:

$$
C_{11}>0,\left(C_{11}-C_{12}\right)>0, C_{44}>0,\left(C_{11}+C_{12}\right) C_{33}>2 C_{13}^{2}
$$


For zinc-blende GaN, which has a cubic crystal structure, the independent elastic constants are $C_{11}, C_{44}$ and $C_{12}$, and its stability requires [44]:

$$
\left(C_{11}+2 C_{12}\right)>0, C_{44}>0,\left(C_{11}-C_{12}\right)>0
$$

The elastic constants calculated under pressures of 0 and 40 GPa are given in Table 2, where results obtained are consistent with previous experimental data and calculation values, demonstrating the effectiveness of the calculations performed in this work. The calculation results show that, under the pressure from 0 to $40 \mathrm{GPa}$, elastic constants of the two GaN crystals satisfy relevant stability criteria, thereby, indicating that the two GaN crystals were mechanically stable under the various pressures applied.

Bulk modulus is a parameter about how incompressible/resistant to compression that substance is. It can be defined as the ratio of the increase of infinitesimal pressure to the corresponding decrease of volume and can be calculated within the Voigt-Reuss scheme [45]:

$$
\begin{aligned}
& B_{V}=\frac{1}{9}\left[C_{11}+C_{22}+C_{33}+2\left(C_{12}+C_{13}+C_{23}\right)\right] \\
& B_{R}=\left[S_{11}+S_{22}+S_{33}+2\left(S_{12}+S_{13}+S_{23}\right)\right]^{-1}
\end{aligned}
$$

where $B_{V}$ and $B_{R}$ denote the upper and lower bounds of the bulk moduli of the polycrystalline aggregate, respectively. The shear modulus of polycrstals can be expressed as Equations (5) and (6):

$$
\begin{gathered}
G_{V}=\frac{1}{15}\left[\left(C_{11}+C_{22}+C_{33}\right)-\left(C_{12}+C_{13}+C_{23}\right)+3\left(C_{44}+C_{55}+C_{66}\right)\right] \\
G_{R}=15\left[4\left(S_{11}+S_{22}+S_{33}\right)-4\left(S_{12}+S_{13}+S_{23}\right)+3\left(S_{44}+S_{55}+S_{66}\right)\right]^{-1}
\end{gathered}
$$

where $G_{V}$ and $G_{R}$ are the upper and lower bounds of shear moduli, respectively. Then, the effective bulk and shear moduli, $B$ and $G$, can be predicted according to the Voigt-Reuss-Hill approximation [45]:

$$
\begin{aligned}
& B=\left(B_{V}+B_{R}\right) / 2 \\
& G=\left(G_{V}+G_{R}\right) / 2
\end{aligned}
$$

Moreover, the elastic modulus $(E)$ of the two GaN polycrystals can be obtained according to Equation (9), and Poisson's ratio (v) can be gotten by Equation (10):

$$
\begin{aligned}
& E=\frac{9 B G}{3 B+G} \\
& v=\frac{3 B-2 G}{6 B+2 G}
\end{aligned}
$$

The calculated mechanical properties for the two GaN polycrystals at pressures of 0 and $40 \mathrm{GPa}$ are listed in Table 3. Clearly, at zero pressure, the three values of $B, G$ and $E$ of the two GaN are similar. However, the mechanical properties of GaN crystals are markedly different at the two pressures 0 and $40 \mathrm{GPa}$. To further explore the influence of hydrostatic pressure on the mechanical behaviour of the two GaN crystals, the pressure dependences of $B, G, B / G$ and $E$ of the two $G a N$ crystals were calculated, see Figure 3. Calculation results display that the bulk modulus $B$ of $\mathrm{GaN}$ increased dramatically with the increase in pressure in a linear fashion. The bulk moduli of wurtzite and zinc-blende GaN polycrystals increased from 195.17 and $200.98 \mathrm{GPa}$ to 355.27 and $358.60 \mathrm{GPa}$, respectively. Meanwhile, the bulk moduli of the two GaN polycrystals at different pressures were quite similar, see Figure $3 a$. The shear moduli of wurtzite and zinc-blende GaN decreased with the increase in pressure, see Figure $3 \mathrm{~b}$. As a result, the ratio $B / G$ of the two crystals increased significantly with the increase in 
pressure, as presented in Figure 3c. Moreover, the results show that with the increase of pressure, the elastic moduli of the two GaN polycrystals may increase at the beginning, while they may decrease as the pressure rises from 20 to $40 \mathrm{GPa}$, as illustrated in Figure 3d.

Table 2. Calculated elastic constant $C_{i j}(\mathrm{GPa})$ under pressures 0 and $40 \mathrm{GPa}$.

\begin{tabular}{|c|c|c|c|c|c|c|c|}
\hline Phases & $C_{11}=C_{22}$ & $C_{12}$ & $C_{22}=C_{23}$ & $C_{33}$ & $C_{44}=C_{55}$ & $C_{66}$ & Note \\
\hline \multirow{4}{*}{ Wurtzite GaN } & 364.19 & 125.99 & 364.19 & 413.68 & 97.04 & 119.10 & $\mathrm{LDA}, 0 \mathrm{GPa}^{\mathrm{a}}$ \\
\hline & 509.98 & 302.12 & 509.98 & 585.85 & 87.06 & 103.93 & $\mathrm{LDA}, 40 \mathrm{GPa}^{\mathrm{a}}$ \\
\hline & 396 & 144 & 396 & 392 & 91 & 126 & LDA, 0GPa [46] \\
\hline & $390 \pm 15$ & $145 \pm 20$ & $390 \pm 15$ & $398 \pm 20$ & $105 \pm 10$ & $123 \pm 10$ & Exp. 0 GPa [47] \\
\hline \multirow{4}{*}{ Zinc-blende GaN } & 287.07 & 157.94 & 287.07 & 287.07 & 167.21 & 167.21 & $\mathrm{LDA}, 0 \mathrm{GPa}^{\mathrm{a}}$ \\
\hline & 426.42 & 324.69 & 426.42 & 426.42 & 171.96 & 171.96 & $\mathrm{LDA}, 40 \mathrm{GPa}^{\mathrm{a}}$ \\
\hline & 285 & 161 & 285 & 285 & 149 & 149 & LDA, 0 GPa [48] \\
\hline & 293 & 159 & 293 & 293 & 155 & 155 & LDA, 0 GPa [49] \\
\hline
\end{tabular}

a This work.

Table 3. Mechanical properties of GaN polycrystals.

\begin{tabular}{|c|c|c|c|c|c|c|}
\hline Structure & $B, \mathrm{GPa}$ & $G, \mathrm{GPa}$ & $E, \mathrm{GPa}$ & $v$ & $B / G$ & Note \\
\hline \multirow{5}{*}{ Wurtzite GaN } & 195.17 & 116.35 & 291.18 & 0.25 & 1.68 & $\mathrm{LDA}, 0 \mathrm{GPa}{ }^{\mathrm{a}}$ \\
\hline & 355.27 & 106.58 & 290.67 & 0.36 & 3.33 & $\mathrm{LDA}, 40 \mathrm{GPa}^{\mathrm{a}}$ \\
\hline & 170 & 103 & 257 & 0.248 & 1.65 & GGA, 0 GPa [50] \\
\hline & 208 & 115 & 291 & 0.267 & 1.81 & LDA, $0 \mathrm{GPa}[50]$ \\
\hline & 170 [51] 188 [52] & $116[53]$ & $295 \pm 3[54]$ & $0.23 \pm 0.06[55]$ & & Exp., $0 \mathrm{GPa}$ \\
\hline \multirow{5}{*}{ Zinc-blende GaN } & 200.98 & 113.56 & 286.67 & 0.26 & 1.77 & $\mathrm{LDA}, 0 \mathrm{GPa}{ }^{\mathrm{a}}$ \\
\hline & 358.60 & 104.31 & 285.26 & 0.37 & 3.44 & $\mathrm{LDA}, 40 \mathrm{GPa}^{\mathrm{a}}$ \\
\hline & 184.41 & 105.30 & 265.38 & 0.260 & 1.75 & GGA, 0 GPa [56] \\
\hline & 184 & 106 & 267 & 0.258 & 1.74 & GGA, 0 GPa [57] \\
\hline & 203.7 & 110.71 & 281.18 & 0.27 & 1.84 & Exp., 0 GPa [58] \\
\hline
\end{tabular}
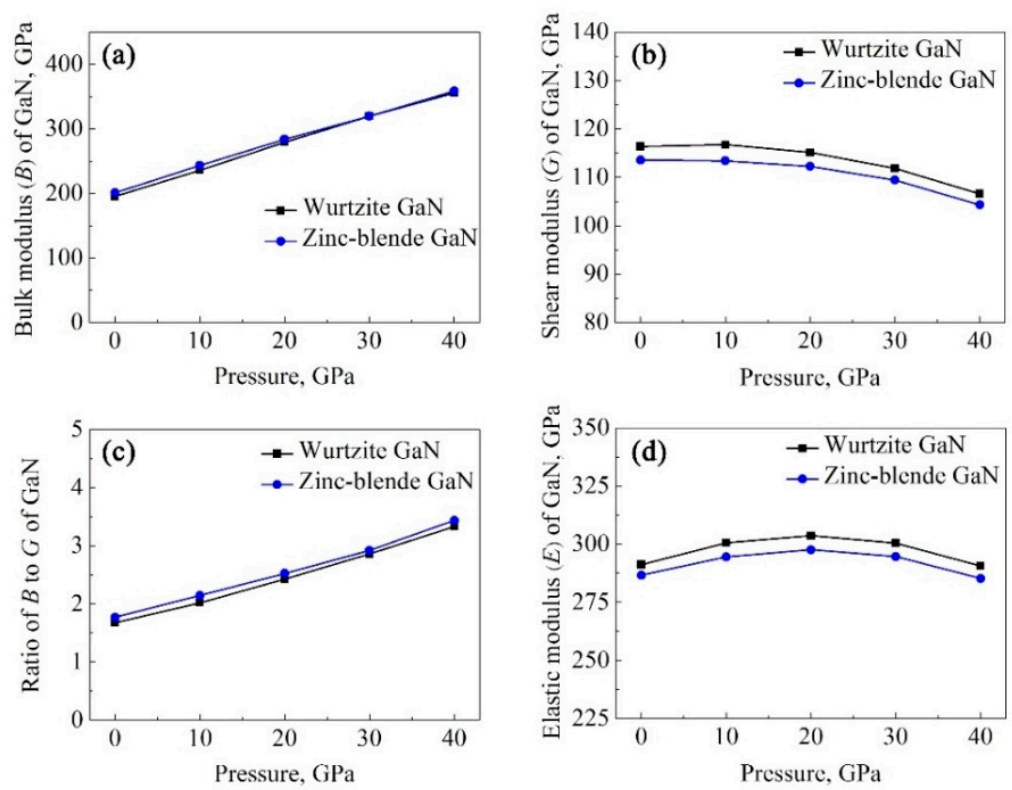

Figure 3. Hydrostatic pressure dependences of bulk modulus $B(\mathbf{a})$, shear modulus $G(\mathbf{b}), B / G(\mathbf{c})$ and elastic modulus $E(\mathbf{d})$ of GaN crystals.

It was reported that the directionality of covalent bonds can be characterized by Poisson's ratio $v$, whose value for covalent materials is small (about 0.1 ); the typical value is 0.25 for ionic materials [59]. In this study, the calculated values of Poisson's ratios were 0.25 and 0.26 for the wurtzite and zinc-blende GaN polycrystals at zero pressure, respectively, see Table 3, indicating strong ionic 
bonds in the two GaN crystals. In addition, Poisson's ratio $(v)$ can be used to evaluate material stability during shear deformation and a smaller value implies a larger stiffness. Thus, wurtzite GaN is more stable at zero pressure. Moreover, according to the Pugh criterion, if the ratio of bulk modulus to shear modulus $B / G$ is larger than 1.75 , ductile fracture occurs; otherwise, the fracture is in a brittle mode [60]. Clearly, at zero pressure, the ratio $B / G$ is 1.68 for wurtzite $G a N$, which is less than 1.75, as given in Table 3. The ratio of zinc-blende $\mathrm{GaN}$ is 1.77 , which is larger than that of wurtzite GaN and close to 1.75. Furthermore, the nature of the bonding, such as brittleness or ductility, can also be evaluated from the Cauchy pressure [61]. A positive Cauchy pressure indicates damage tolerance and ductility, while a negative value reveals brittleness; it is supposed that the hexagonal structure is intrinsically brittle [61]. For cubic zinc-blende $G a N$, the Cauchy pressure is defined as $C_{12}-C_{44}$. At zero pressure, Cauchy pressure is negative according to the data listed in Table 2, thus indicating that the zinc-blende $\mathrm{GaN}$ would also exhibit brittle behaviour at zero pressure. When the pressure increases to $40 \mathrm{GPa}$, the calculated Poisson's ratios of wurtzite and zinc-blende $\mathrm{GaN}$ polycrystals are 0.36 and 0.37 , respectively; meanwhile, values of $B / G$ rise to 3.33 and 3.44 , respectively, revealing that the two GaN polycrystals may exhibit ductile behaviours under high stresses.

\subsection{Elastic Anisotropy}

The regular arrangements of atoms in crystals determine the essential elastic anisotropy, which has a significant influence on the mechanical properties of materials, such as crack growth, anisotropic elastic deformation and elastic instability. In this study, the anisotropy factor $(A)$ is employed to evaluate the anisotropic degree of the two GaN single crystal [62]. If $A$ in a crystal plane is close to 1 , then the corresponding mechanical property is prone to isotropy. Results in Table 4 show that, at zero pressure, wurtzite GaN displays strong anisotropic characteristics in the planes containing the [001] axis, and zinc-blende GaN shows obvious anisotropy at planes $\{100\}$ and $\{110\}$. In general, the increase in pressure raises the elastic anisotropy of GaN crystals.

Table 4. Anisotropy factors for two GaN single crystals.

\begin{tabular}{cccc}
\hline Structure & Symmetry & \multicolumn{2}{c}{ Anisotropy Factor $A$} \\
\hline \multirow{2}{*}{ Wurtzite GaN } & Planes containing the [001] axis & $C_{44}\left(C_{11}+2 C_{13}+C_{33}\right) /\left(C_{11} C_{33}-C_{13}^{2}\right)$ & $0.653,0 \mathrm{GPa}$ \\
& & $2.582,40 \mathrm{GPa}$ \\
\hline & $\{100\}$ & $2 C_{44} /\left(C_{11}-C_{12}\right)$ & $2.560,0 \mathrm{GPa}$ \\
Zinc-blendeGaN & $\{110\}$ & $C_{44}\left(C_{L}+2 C_{12}+C_{11}\right) /\left(C_{L} C_{11}-C_{12}^{2}\right)^{*}$ & $\begin{array}{c}3.381,40 \mathrm{GPa} \\
1.909,0 \mathrm{GPa} \\
2.180,40 \mathrm{GPa}\end{array}$ \\
\hline
\end{tabular}

${ }^{*}$ For cubic crystals $C_{L}=C_{66}+\left(C_{11}+C_{12}\right) / 2$.

To further explore the anisotropic behaviours of the two GaN single crystals, the three-dimensional (3D) surface, which can reveal the elastic anisotropy, was analysed. The 3D surface reflects the variation in the elastic modulus $E$ of the crystal in different crystal directions, and the direction dependence of the elastic modulus can be expressed as [44]:

$$
\begin{aligned}
1 / E=S_{11} l_{1}^{4}+2 S_{12}\left(l_{1} l_{2}\right)^{2}+2 S_{13}\left(l_{1} l_{3}\right)^{2}+2 S_{14}\left(l_{1}^{2} l_{2} l_{3}\right)+2 S_{15}\left(l_{3} l_{1}^{3}\right)+2 S_{16}\left(l_{1}^{3} l_{2}\right) \\
+S_{22} l_{2}^{4}+2 S_{23}\left(l_{2} l_{3}\right)^{2}+2 S_{24}\left(l_{2}^{3} l_{3}\right)+2 S_{25}\left(l_{1} l_{2}^{2} l_{3}\right)+2 S_{26}\left(l_{1} l_{2}^{3}\right) \\
+S_{33} l_{3}^{4}+2 S_{34}\left(l_{2} l_{3}^{3}\right)+2 S_{35}\left(l_{1} l_{3}^{3}\right)+2 S_{36}\left(l_{1} l_{2} l_{3}^{2}\right) \\
+S_{44}\left(l_{2} l_{3}\right)^{2}+2 S_{45}\left(l_{1} l_{2} l_{3}^{2}\right)+2 S_{46}\left(l_{1} l_{3} l_{2}^{2}\right) \\
+S_{55}\left(l_{1} l_{3}\right)^{2}+2 S_{56}\left(l_{2} l_{3} l_{1}^{2}\right) \\
+ \\
+S_{66}\left(l_{1} l_{2}\right)^{2}
\end{aligned}
$$

where $S_{i j}$ denotes the compliance coefficient, which can be obtained from the inverse matrix of matrix $C_{i j}$ (i.e., $S_{i j}=C_{i j}$ ), and $l_{1}, l_{2}$ and $l_{3}$ are three direction cosines concerning the $a, b$ and $c$ axes, respectively. Considering the crystal symmetries of hexagonal wurtzite and cubic zinc-blende 
crystals, Equation (11) for wurtzite and zinc-blende GaN single crystals can be simplified as Equations (12) and (13), respectively:

$$
\begin{aligned}
& E=1 /\left[\left(1-l_{3}^{2}\right)^{2} S_{11}+l_{3}^{4} S_{33}+l_{3}^{2}\left(1-l_{3}^{2}\right)\left(2 S_{13}+S_{44}\right)\right] \\
& E=1 /\left[S_{11}+2\left(S_{11}-S_{12}-0.5 S_{44}\right)\left(l_{1}^{2} l_{2}^{2}+l_{2}^{2} l_{3}^{2}+l_{3}^{2} l_{1}^{2}\right)\right]
\end{aligned}
$$

Direction dependences of elastic moduli are displayed in Figure 4. For the wurtzite GaN single crystal, the curve shape at (001) plane is almost circular. However, at planes containing a [001] axis, the shape is far from a circle; therefore, wurtzite GaN exhibits an isotropic character at the (001) plane, while showing obvious anisotropy at planes (100) and (010). Meanwhile, for wurtzite GaN, the maximum value is located at the [001] axis. Under a high pressure of $40 \mathrm{GPa}$, the anisotropy of wurtzite GaN at planes containing the [001] axis increases significantly from 0.65 at zero pressure to 0.58 , and the maximum elastic modulus along the [001] axis increases from 380.13 to $435.67 \mathrm{GPa}$, as shown in Figure $4 \mathrm{a}-\mathrm{c}$. For zinc-blende GaN, the minimum elastic modulus is along the three axes (i.e., $<100>$ directions) and the value is $174.96 \mathrm{GPa}$ at zero pressure. However, this decreases to $145.71 \mathrm{GPa}$ under a 40 GPa pressure. The maximum elastic moduli are located at directions $\{111\}$, and the values are 392.7 and $444.7 \mathrm{GPa}$ at zero and $40 \mathrm{GPa}$ pressures, respectively. Meanwhile, the anisotropies at planes $\{100\}$ and $\{110\}$ increase from 2.56 and 1.91 to 3.38 and 2.18, respectively, with the pressure changes from 0 to $40 \mathrm{GPa}$.

Furthermore, the shear moduli of wurtzite and zinc-blende GaN single crystals can be calculated by Equation (14) [63] and Equations (15)-(17), respectively:

$$
\begin{gathered}
\frac{1}{G}=S_{55}+\left(S_{11}-S_{12}-\frac{S_{44}}{2}\right)\left(1-l_{3}^{2}\right)+2\left(S_{11}+S_{33}-2 S_{13}-S_{44}\right)\left(1-l_{3}^{2}\right) l_{3}^{2} \\
\frac{1}{G}=\left(S_{44}+4 S_{0} J\right) \\
S_{0}=S_{11}-S_{12}-\frac{1}{2} S_{44} \\
J=\sin ^{2} \theta \cdot \cos ^{2} \theta+0.125 \cdot \sin ^{4} \theta(1-\cos 4 \phi)
\end{gathered}
$$

where $\theta$ and $\varphi$ are the two Euler angles in direction cosines.

Figure 5 shows the calculated direction dependences of shear moduli. For wurtzite $\mathrm{GaN}$, the curve shape at the (001) plane is a circle at both 0 and $40 \mathrm{GPa}$, while it is clearly not at the planes containing c or [001] axis. Accordingly, the shear deformation of wurtzite GaN may be isotropic at the (001) crystal plane and the values are 97.03 and $87.06 \mathrm{GPa}$ under pressures 0 and $40 \mathrm{GPa}$, respectively. However, at planes containing the [001] axis, anisotropic characteristics are observed, and the maximum and minimum values are 124.23 and $97.03 \mathrm{GPa}$ at zero pressure, respectively, while they decrease to 114.72 and $87.06 \mathrm{GPa}$, respectively, at a pressure of $40 \mathrm{GPa}$. For zinc-blende GaN single crystal, it was revealed that shear moduli along directions [100], [010] and [001] are maximal and the value is 167.21 GPa, while the minimum value is located at crystal orientation family $<111>$ and the value is $81.18 \mathrm{GPa}$, as shown in Figure 5d. Additionally, for zinc-blende GaN, obvious anisotropic characteristics were observed. At zero pressure, the maximum and minimum values of shear moduli at planes $\{100\}$ are 167.22 and 93.16 GPa, respectively, while they are 171.96 and $78.51 \mathrm{GPa}$, respectively, at apressure of 40GPa, see Figure $5 \mathrm{~d}-\mathrm{f}$. Compared to the directional dependence of elastic modulus, the shear modulus has an inverse trend. For examples, although the elastic modulus at orientation [100] is maximal, the shear modulus at [100] is minimal. Therefore, both elastic and shear moduli of the two GaN single crystals exhibit obvious anisotropy characteristics. Nevertheless, our results show that the bulk moduli of the 
two GaN single crystals are almost isotropic. Bulk moduli of wurtzite and zinc-blende GaN single crystals can be expressed as Equations (18) and (19), respectively:

$$
\begin{gathered}
\frac{1}{B}=\left(S_{11}+S_{12}+S_{13}\right)-\left(S_{11}+S_{12}-S_{13}-S_{33}\right) l_{3}^{2} \\
\frac{1}{B}=\left(S_{11}+2 S_{12}\right)\left(l_{1}^{2}+l_{2}^{2}+l_{3}^{2}\right)
\end{gathered}
$$

The maximum bulk modulus of wurtzite GaN is at crystal directions vertical to orientation [001], and the value is $603.32 \mathrm{GPa}$ at zero pressure. Meanwhile, the minimum value is $576.87 \mathrm{GPa}$ and it is located in the [100] direction, see Figure 6a. In addition, as the applied pressure increased to $40 \mathrm{GPa}$, the maximum value and minimum value of bulk moduli increased to 1111.98 and $1044.08 \mathrm{GPa}$, respectively, as exhibited in Figure 6b. Clearly, the anisotropy of bulk modulus is quite slight for wurtzite $\mathrm{GaN}$, and the influence of pressure on its anisotropy is weak. For zinc-blende GaN, the bulk modulus is totally isotropic and its value is $602.92 \mathrm{GPa}$ at zero pressure, see Figure 6c. In spite of this, the value will rise with increasing pressure, e.g., it is $1075.85 \mathrm{GPa}$ at apressure of $40 \mathrm{GPa}$ (Figure 6d), there is still no anisotropy for bulk modulus.

Furthermore, for wurtzite GaN single crystal, Poisson's ratios at crystallographic planes containing [001] axis, $v(\theta)$, can be calculated with Equation (20) [64], and Poisson's ratios of zinc-blende GaN at the plane ( $h k l)$ can be expressed with Equation (21) [65]:

$$
\begin{gathered}
v(\theta)=\frac{s_{12} \sin ^{2} \theta+s_{13} \cos ^{2} \theta}{s_{11} \sin ^{4} \theta+s_{33} \cos ^{4} \theta+\left(s_{44}+2 s_{13}\right) \sin ^{2} \theta \cos ^{2} \theta} \\
v(h k l, \theta)=\left\{S_{12}+\frac{s_{0}}{h^{2}+k^{2}+l^{2}}\left[\left(\frac{h^{2} l}{\sqrt{h^{2}+k^{2}} \sqrt{h^{2}+k^{2}+l^{2}}} \cos \theta-\frac{h k}{\sqrt{h^{2}+k^{2}}} \sin \theta\right)^{2}+\right.\right. \\
\left.\left.\left(\frac{k^{2} l}{\sqrt{h^{2}+k^{2}} \sqrt{h^{2}+k^{2}+l^{2}}} \times \cos \theta+\frac{h k}{\sqrt{h^{2}+k^{2}}} \sin \theta\right)^{2}+\left(\frac{l \sqrt{h^{2}+k^{2}}}{\sqrt{h^{2}+k^{2}+l^{2}}} \cos \theta\right)^{2}\right]\right\} \\
/\left[-S_{11}+2 S_{0} \frac{(h k)^{2}+(h l)^{2}+(l k)^{2}}{\left(h^{2}+k^{2}+l^{2}\right)^{2}}\right]
\end{gathered}
$$

Figure 7a illustrates the Poisson's ratios of wurtzite GaN at the planes containing the [001] axis, in which the maximum Poisson's ratio is 0.31 at zero pressure and the value is in the orientations vertical to [001] axis. Correspondingly, the minimum value of Poisson's ratio is 0.19 , and the angle between its orientation and direction [001] is $25.8^{\circ}$. When the pressure increased to $40 \mathrm{GPa}$, the maximum and minimum values were 0.488 and 0.271 , respectively, and their locations were the same with those at zero pressure. In addition, the Poisson's ratio along the [001] direction of wurtzite GaN were 0.185 and 0.304 at pressures 0 and $40 \mathrm{GPa}$, respectively. Moreover, Poisson's ratios of zinc-blende GaN at crystal planes (100) and (111) are isotropic, see Figure 7b,c; they were 0.355 and 0.174 , respectively, at zero pressure and 0.432 and 0.293 , respectively, at apressure of $40 \mathrm{GPa}$. However, for zinc-blende GaN, the Poisson's ratio at crystal plane (110) showed obvious anisotropy, see Figure 7d, where the maximum values, $0.608(0 \mathrm{GPa})$ and $0.872(40 \mathrm{GPa})$, were located at the [001] and [001] directions. In addition, the minimum values were $0.104(0 \mathrm{GPa})$ and 0.145 (40 GPa) (actually, -0.104 and -0.145 ); these are in the orientations [ $[\overline{1} 10]$ or $[1 \overline{1} 0]$ at zero pressure. 
(a)

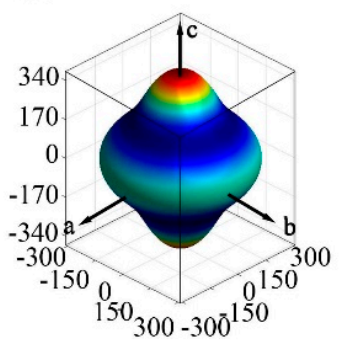

(d)

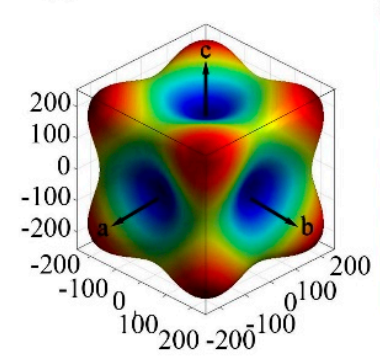

(b)

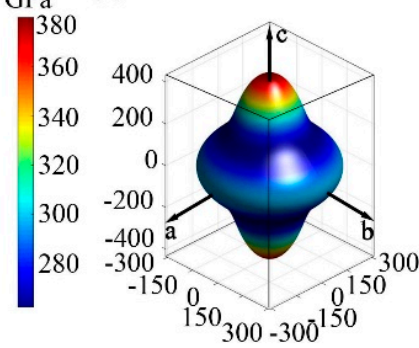

(e)

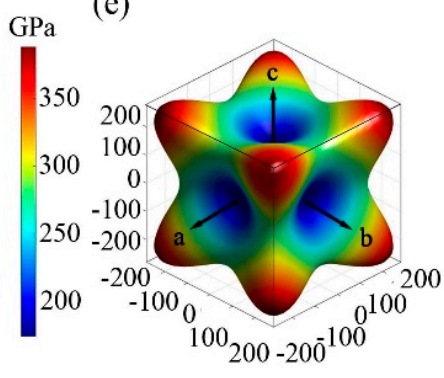

(c) - Planes containing [001] axis, $0 \mathrm{GPa}$

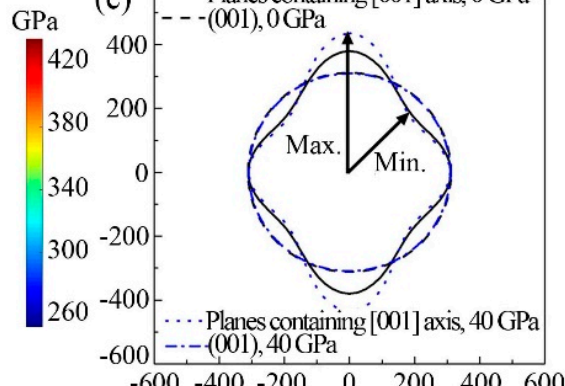

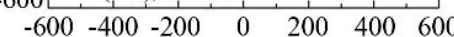

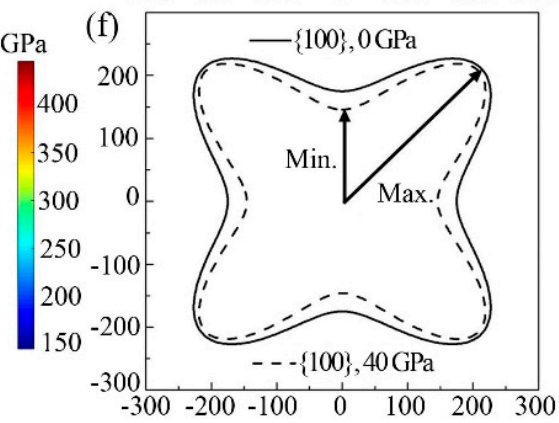

Figure 4. Direction dependences of the elastic moduli of GaN single crystals: (a) wurtzite GaN, 0 GPa; (b) wurtzite GaN, $40 \mathrm{GPa}$; (c) projections on main crystal planes, wurtzite GaN; (d) zinc-blende GaN, $0 \mathrm{GPa}$; (e) zinc-blende GaN, $40 \mathrm{GPa}$; (f) projections on main crystal planes, zinc-blende GaN.

(a)

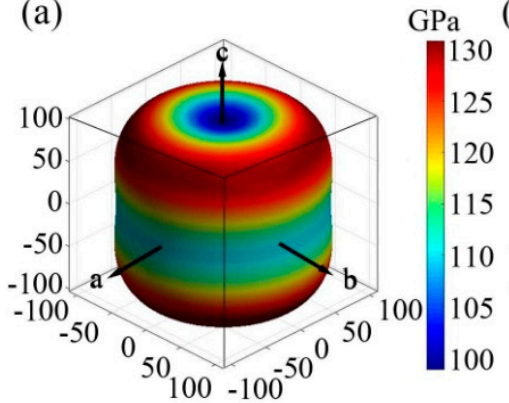

(d)

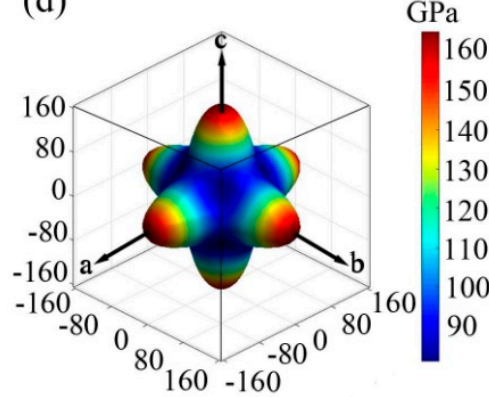

(b)

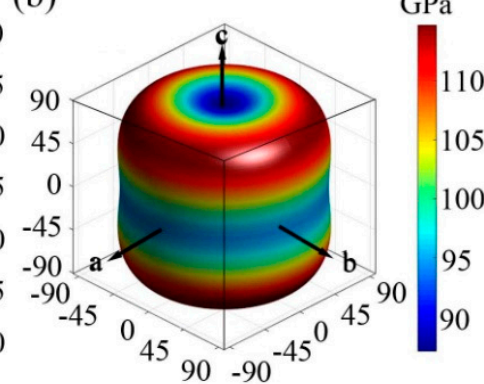

(e)

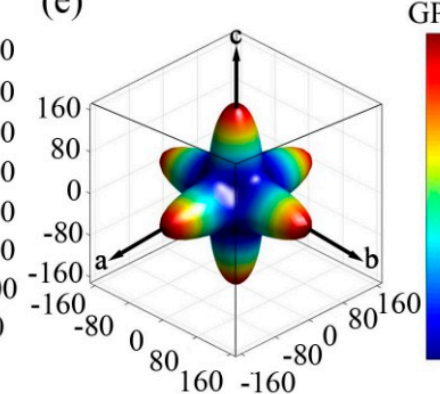

(c) Planes comtaining [001] axis, $0 \mathrm{GPa}$

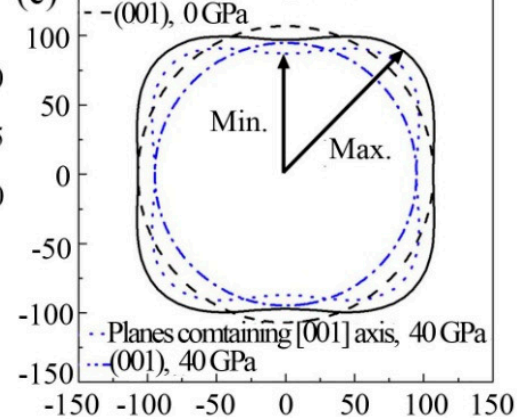

$\begin{array}{lllllll}-150 & -100 & -50 & 0 & 50 & 100 & 150\end{array}$

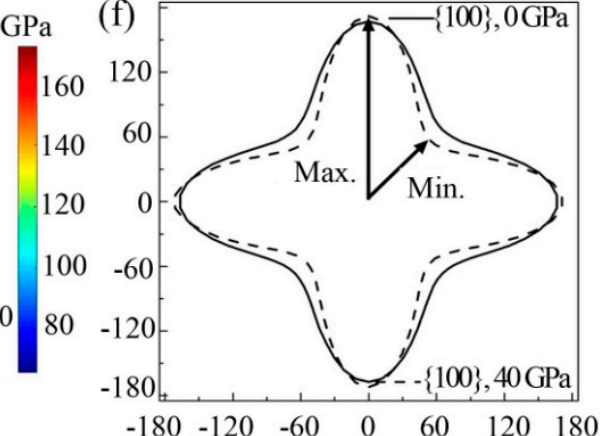

Figure 5. Direction dependences of the shear moduli of: (a) wurtzite GaN, $0 \mathrm{GPa}$; (b) wurtzite $\mathrm{GaN}, 40 \mathrm{GPa}$; (c) projections on main crystal planes, wurtzite GaN; (d) zinc-blende GaN, $0 \mathrm{GPa}$; (e) zinc-blende GaN, $40 \mathrm{GPa}$; (f) projections on main crystal planes, zinc-blende GaN. 

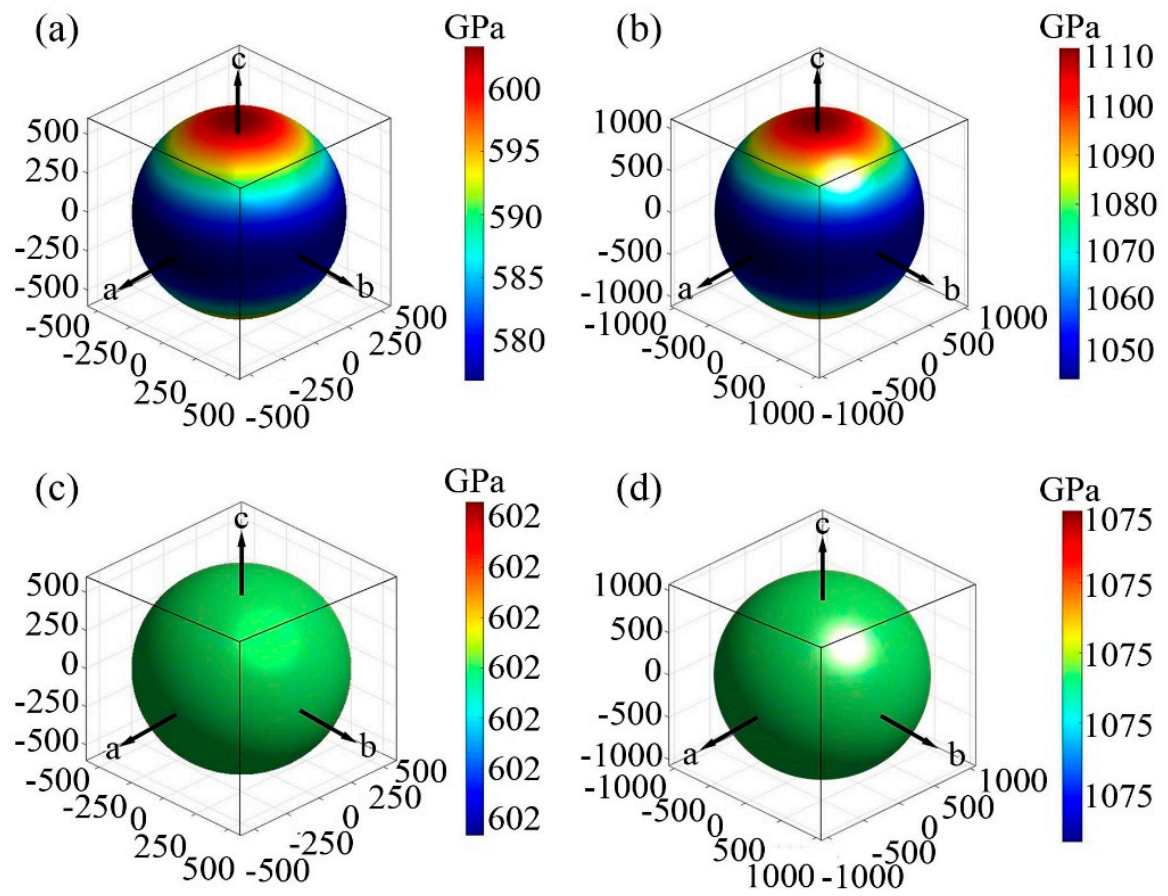

Figure 6. Direction dependences of the bulk moduli of: (a) wurtzite GaN, 0 GPa; (b) wurtzite GaN, 40 GPa; (c) zinc-blende GaN, 0 GPa; (d) zinc-blende GaN, 40 GPa.
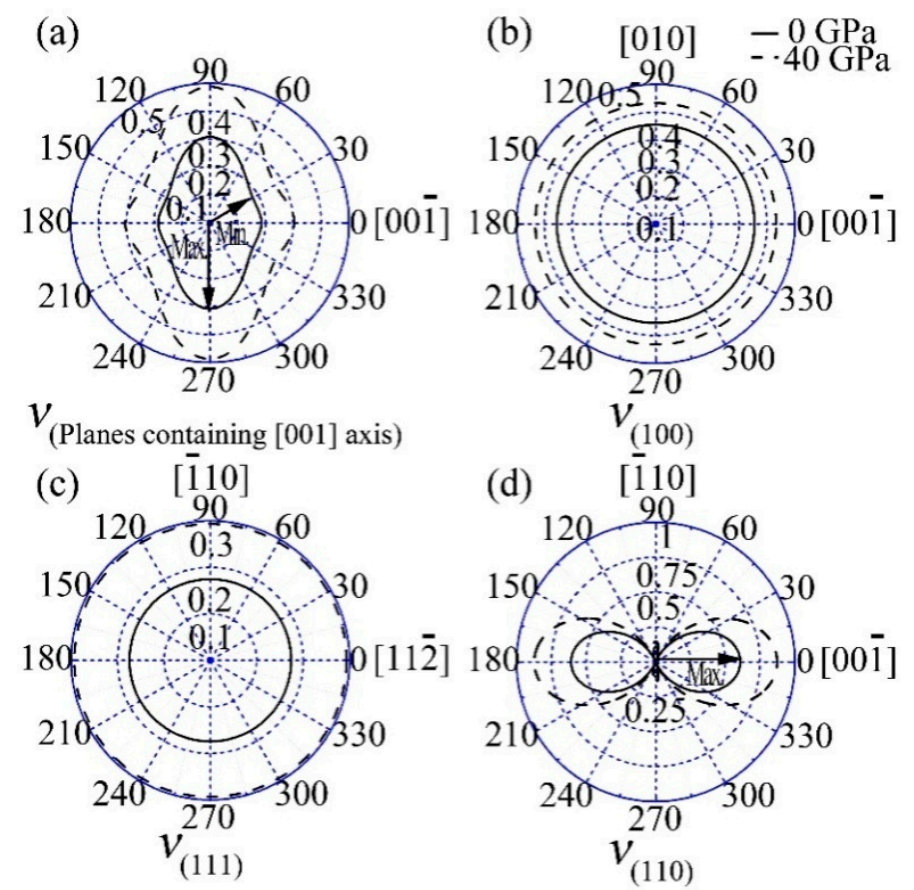

Figure 7. Poisson's ratios of wurtzite GaN at planes containing [001] ([0001]) axis (a); and Poisson's ratio of zinc-blende GaN at three crystal planes (100) (b), (111) (c) and (110) (d).

\subsection{Electronic Properties}

Band gap is a basic and critical physical parameter that determines the electronic properties of semiconductor materials. It has been reported that the band gap of wurtzite $\mathrm{GaN}$ is $3.5 \mathrm{eV}$ [58] and $3.1 \mathrm{eV}$ for zinc-blende GaN [66]. The influence of pressure on the electronic properties of the two GaN crystals was evaluated. In this study, a HSE06 scheme [67] was used for the calculation of the band gap. Results show that, at zero pressure, the calculated band gaps of wurtzite and zinc-blende 
GaN crystals were 3.62 and $3.01 \mathrm{eV}$, respectively, which are similar to previous experimental values. Figure 8 displays the calculated band gaps of the two GaN crystals at different hydrostatic pressures. Clearly, both crystal structure and pressure have obvious influence on the band gap, and the band gap of wurtzite GaN is much larger at the same pressure. Generally, the band gaps of the two GaN compounds increased linearly with the increase of hydrostatic pressures; the lines in Figure 8 can be analysed by the following equation [68]:

$$
E_{g}(P)=E_{g}(0)+k P
$$

where $E_{g}(P)$ is the band gap, $E_{g}(0)$ is the band gap at zero pressure, $k$ is the pressure coefficient and $k=\mathrm{d} E_{g}(P) / \mathrm{d} P$, and $P$ is the applied hydrostatic pressure.

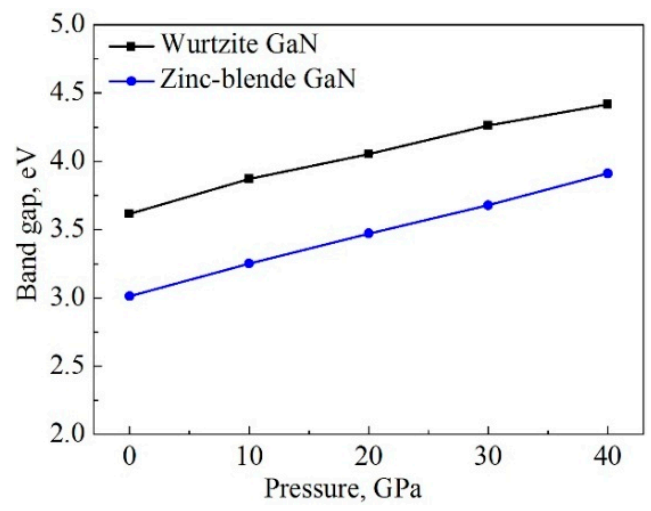

Figure 8. Band gaps of two GaN structures at different pressures.

According to our simulation, the $k$ values for wurtzite and zinc-blende GaN crystals were 20.8 and 22.6, respectively. As the pressure rose from 0 to $40 \mathrm{GPa}$, the band gaps of wurtzite and zinc-blende $\mathrm{GaN}$ crystals increased from 3.62 and $3.01 \mathrm{eV}$ to 4.41 and $3.91 \mathrm{eV}$, respectively. The band structures at 0 and $40 \mathrm{GPa}$ are exhibited in Figure 9. It was revealed that the upper limit of valence band and the lower limit of conduction band are located at $\mathrm{G}$ point, and they are direct gap semiconductor materials.
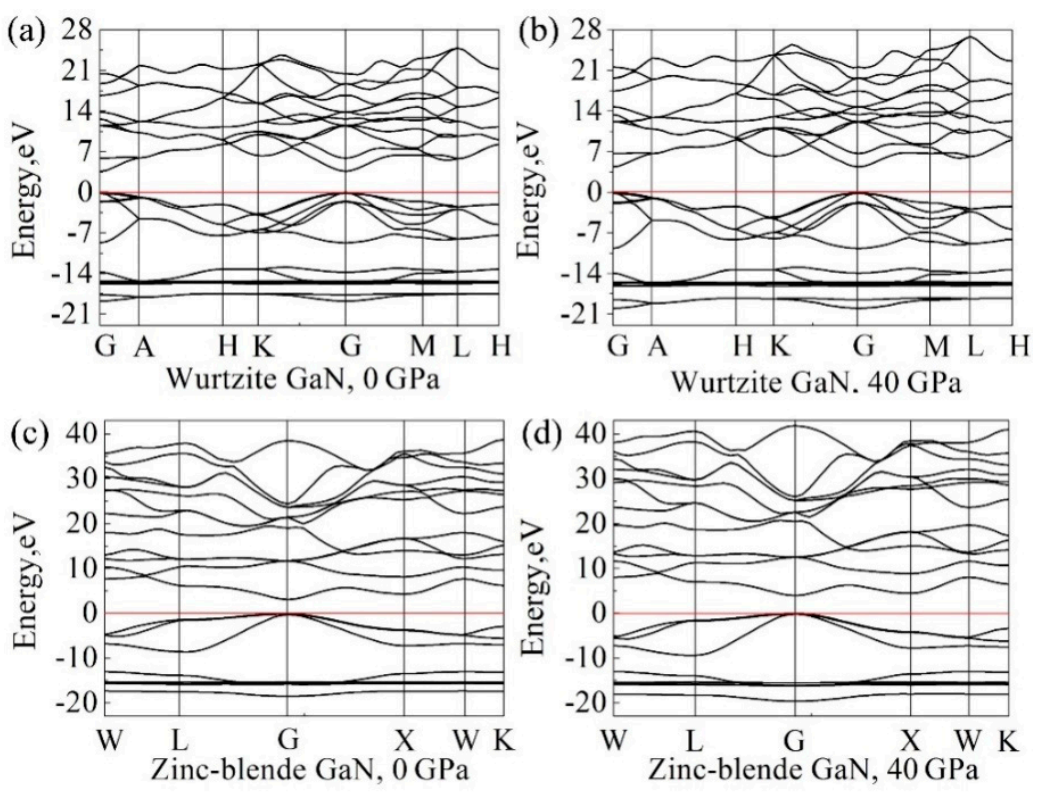

Figure 9. Band structures: (a) wurtzite GaN, $0 \mathrm{GPa}$; (b) wurtzite GaN, $40 \mathrm{GPa}$; (c) zinc-blende GaN, 0 GPa; (d) zinc-blende GaN, 40 GPa. 
To further understand the pressure effect on the band structure, the density of states (DOSs) of GaN crystals were also analysed in this study. Figure 10 reveals DOSs near band gaps. Clearly, the two $\mathrm{GaN}$ crystals show similar atomic bonding and hybridization behaviour between $\mathrm{Ga}$ and $\mathrm{N}$ atoms. In the energy band between -10 and $0 \mathrm{eV}$, p orbits of $\mathrm{N}(\mathrm{N}-\mathrm{p})$ contribute greatly to the DOSs. However, for the energy band in the conduction band near the band gap, e.g., the energies between 1.66 to $20 \mathrm{eV}$ in the wurtzite GaN, the hybridization between N-p and Ga-s/Ga-p induced the formation of DOSs. Moreover, as the pressure rose from 0 to $40 \mathrm{GPa}$, DOSs above $0 \mathrm{eV}$ moved towards the right side, as shown in Figure 10. For example, under zero pressure, peaks A, B and C of DOSs for wurtzite and zinc-blende $\mathrm{GaN}$ were located at 6.65 and $6.25 \mathrm{eV}$, respectively, while their locations were 7.36 and $7.28 \mathrm{eV}$, respectively, at $40 \mathrm{GPa}$. Consequently, band gaps of GaN crystals increased with the increasing pressure.

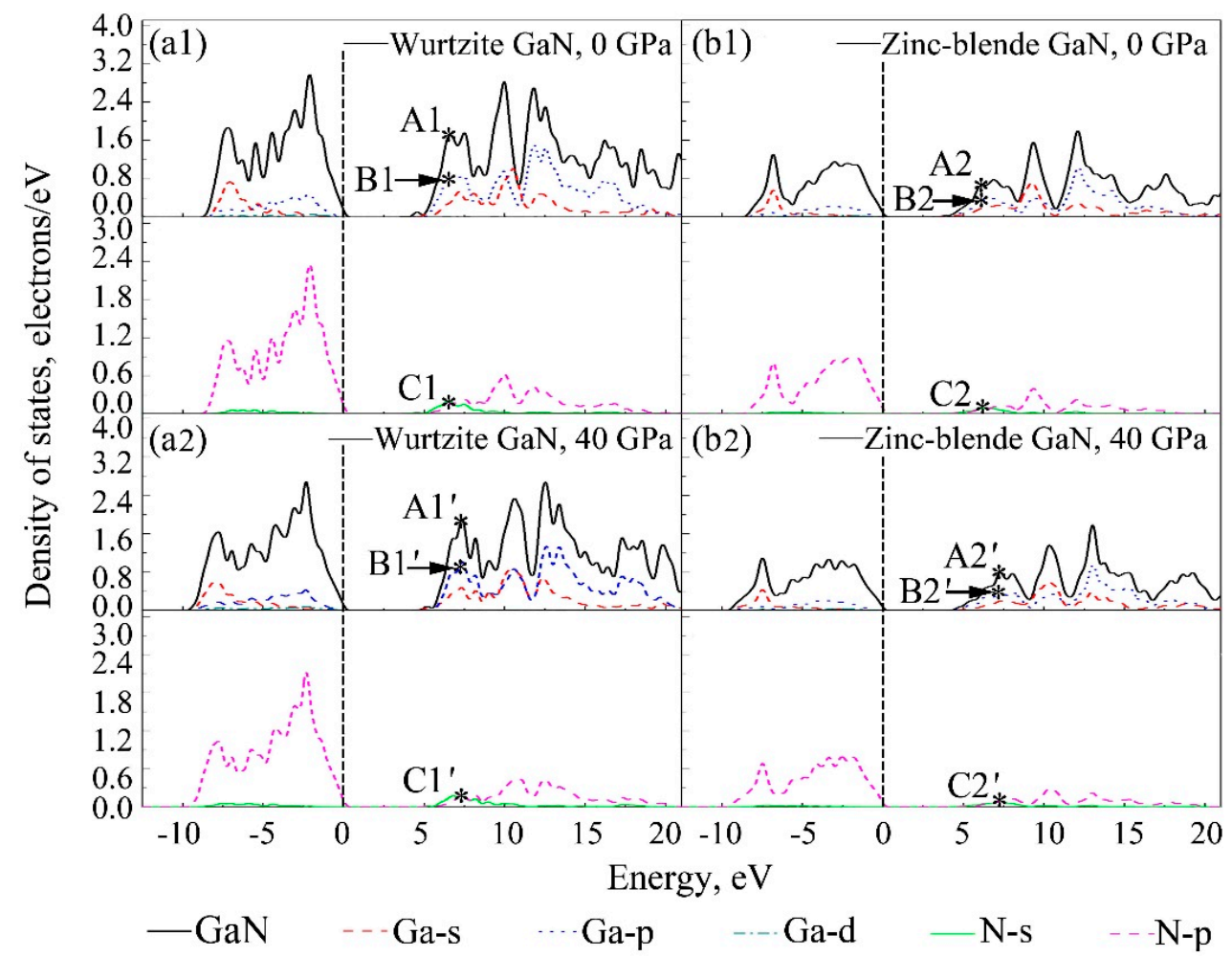

Figure 10. Pressures versus density of states (DOSs) for: (a1) wurtzite GaN, 0 GPa; (a2) wurtzite GaN, 40 GPa; (b1) zinc-blende GaN, 0 GPa, (b2) zinc-blende GaN, 40 GPa.

\section{Conclusions}

The mechanical and electronic properties of two GaN crystals under different hydrostatic pressures were investigated through first-principles calculation, and the following conclusions were drawn:

(1) The lattice constants and elastic constants of wurtzite and zinc-blende GaN crystals calculated by both GGA and LDA match well with the experimental values. The structures of the two GaN crystals are stable under the pressure up to $40 \mathrm{GPa}$.

(2) Bulk moduli increase while shear moduli decrease with the increase in pressure, resulting in the significant increase of the ratios of bulk moduli to shear moduli for the two GaN crystals. The two GaN crystals are brittle materials at zero pressure, while they exhibit ductile behaviours under high stresses.

(3) Increase in pressure raises the elastic anisotropy of GaN single crystals, and the anisotropy factors of the two GaN crystals are quite different. Wurtzite GaN exhibits an isotropic character of 
elastic modules at the (001) plane, while showing obvious anisotropy at planes (100) and (010). The maximum value is located at the [001] axis. Zinc-blende GaN shows the anisotropic elastic modulus in all planes, and the minimum and maximum values are in the directions $<100>$ and $<111>$, respectively.

(4) Compared to the obvious directional dependences of elastic moduli at different pressures, the shear modulus has an inverse trend for the two GaN single crystals. There is hardly any anisotropy for bulk modulus, especially for that of zinc-blende GaN.

(5) The two GaN crystals show similar atomic bonding and hybridization behaviour between Ga and $\mathrm{N}$ atoms. The band gaps of GaN crystals increase with increasing pressure, and zinc-blende GaN has a larger pressure coefficient.

Author Contributions: H.Q. and P.Z. conceived and designed the research; H.Q., T.K., X.L. and W.L. performed the first principles calculation; H.Q. wrote the manuscript; J.X., D.Y. and G.Z. reviewed and edited the manuscript. All authors read and approved the final manuscript.

Funding: this work was funded by the National Natural Science Foundation of China (No. 51505095 and 61764002), the Guangxi Natural Science Foundation (No. 2016GXNSFBA380114 and 2017GXNSFAA198282), the Guangxi Colleges and Universities Program of Innovative Research Team and Outstanding Talent, and the Innovation Project of GUET Graduate Education (No. 2018YJCX04).

Conflicts of Interest: The authors declare no conflict of interest.

\section{References}

1. Nakamura, S. The roles of structural imperfections in InGaN-based blue light-emitting diodes and laser diodes. Science 1998, 281, 956-961. [CrossRef]

2. Wang, X.; Chang, B.; Ren, L.; Gao, P. Influence of the p-type doping concentration on reflection-mode GaN photocathode. Appl. Phys. Lett. 2011, 98, 082109. [CrossRef]

3. Chen, J.; Xue, C.; Zhuang, H.; Yang, Z.; Qin, L.; Li, H.; Huang, Y. Catalytic synthesis and optical properties of large-scale GaN nanorods. J. Alloy Compd. 2009, 468, L1-L4. [CrossRef]

4. Paskova, T.; Hanser, D.A.; Evans, K.R. Gan substrates for III-Nitride devices. Proc. IEEE 2010, 98, $1324-1338$. [CrossRef]

5. Zhuang, D.; Edgar, J. Wet etching of GaN, AlN, and SiC: A review. Mater. Sci. Eng. R Rep. 2005, 48, 1-46. [CrossRef]

6. Dasari, K.; Wu, J.; Huhtinen, H.; Jadwisienczak, W.M.; Palai, R. Growth, luminescence and magnetic properties of GaN:Er semiconductor thin films grown by molecular beam epitaxy. J. Phys. D: Appl. Phys. 2017, 50, 175104. [CrossRef]

7. Gao, X.; Man, B.; Zhang, C.; Leng, J.; Xu, Y.; Wang, Q.; Zhang, M.; Meng, Y. The important role of Ga vacancies in the ferromagnetic GaN thin films. J. Alloys Compd. 2017, 699, 596-600. [CrossRef]

8. Paisley, M.J.; Sitar, Z.; Posthill, J.B.; Davis, R.F. Growth of cubic phase gallium nitride by modified molecular-beam epitaxy. J. Vac. Sci. Technol. A Vac. Surf. Films 1989, 7, 701-705. [CrossRef]

9. Lei, T.; Fanciulli, M.; Molnar, R.J.; Moustakas, T.D.; Graham, R.J.; Scanlon, J. Epitaxial growth of zinc blende and wurtzitic gallium nitride thin films on (001) silicon. Appl. Phys. Lett. 1991, 59, 944-946. [CrossRef]

10. Pankove, J.I. Perspective on gallium nitride. MRS Online Proc. Libr. Arch. 1989, 162. [CrossRef]

11. Zywietz, T.K.; Neugebauer, J.; Scheffler, M. The adsorption of oxygen at GaN surfaces. Appl. Phys. Lett. 1999, 74, 1695-1697. [CrossRef]

12. Adelmann, C.; Brault, J.; Mula, G.; Daudin, B.; Lymperakis, L.; Neugebauer, J. Gallium adsorption on (0001) GaN surfaces. Phys. Rev. B 2003, 67, 165419. [CrossRef]

13. Rosa, A.L.; Neugebauer, J.; Northrup, J.E.; Lee, C.D.; Feenstra, R.M. Adsorption and incorporation of silicon at GaN (0001) surfaces. Appl. Phys. Lett. 2002, 80, 2008-2010. [CrossRef]

14. Lee, D.S.; Lee, J.H.; Lee, Y.H.; Lee, D.D. GaN thin films as gas sensors. Sens. Actuators B Chem. 2003, 89, 305-310. [CrossRef]

15. Pearton, S.; Zolper, J.; Shul, R.; Ren, F. GaN: Processing, defects, and devices. J. Appl. Phys. 1999, 86, 1-78. [CrossRef] 
16. Reshchikov, M.A.; Demchenko, D.O.; Usikov, A.; Helava, H.; Makarov, Y. Carbon defects as sources of the green and yellow luminescence bands in undoped GaN. Phys. Rev. B 2014, 90, 235203. [CrossRef]

17. Li, L.; Yu, J.; Hao, Z.; Wang, L.; Wang, J.; Han, Y.; Li, H.; Xiong, B.; Sun, C.; Luo, Y. Influence of point defects on optical properties of GaN-based materials by first principle study. Comp. Mater.Sci. 2017, 129, 49-54. [CrossRef]

18. Li, E.; Wu, B.; Lv, S.; Cui, Z.; Ma, D.; Shi, W. Field emission properties of ge-doped GaN nanowires. J. Alloys Compd. 2016, 681, 324-329. [CrossRef]

19. Kamimura, J.; Bogdanoff, P.; Ramsteiner, M.; Corfdir, P.; Feix, F.; Geelhaar, L.; Riechert, H. P-type doping of GaN nanowires characterized by photoelectrochemical measurements. Nano Lett. 2017, 17, 1529-1537. [CrossRef] [PubMed]

20. Zhang, Y.P.; Zhang, Z.H.; Tan, S.T.; Hernandezmartinez, P.L.; Zhu, B.B.; Lu, S.P.; Kang, X.J.; Sun, X.W.; Demir, H.V. Investigation of p-type depletion doping for InGaN/GaN-based light-emitting diodes. Appl. Phys. Lett. 2017, 110, 033506. [CrossRef]

21. Yang, M.; Chang, B.; Hao, G.; Wang, H.; Wang, M. Optoelectronic properties of GaN, AlN, and GaAlN alloys. Opt. Int. J. Light Electron Opt. 2015, 126, 3357-3361. [CrossRef]

22. Chen, W.H.; Cheng, H.C.; Yu, C.F. The mechanical, thermodynamic, and electronic properties of cubic $\mathrm{Au}_{4} \mathrm{Al}$ crystal via first-principles calculations. J. Alloys Compd. 2016, 689, 857-864. [CrossRef]

23. Guechi, A.; Merabet, A.; Chegaar, M.; Bouhemadou, A.; Guechi, N. Pressure effect on the structural, elastic, electronic and optical properties of the Zintl phase KAsSn, first principles study. J. Alloys Compd. 2015, 623, 219-228. [CrossRef]

24. Liu, X.K.; Zhou, W.; Zheng, Z.; Peng, S.-M. The elastic and thermodynamic properties of $\mathrm{ZrMo}_{2}$ from first principles calculations. J. Alloys Compd. 2014, 615, 975-982. [CrossRef]

25. Wang, S.Q.; Ye, H.Q. A plane-wave pseudopotential study on III-V zinc-blende and wurtzite semiconductors under pressure. J. Phys. Condens. Matter 2002, 14, 9579-9587. [CrossRef]

26. Segall, M.D.; Lindan, P.J.D.; Probert, M.J.; Pickard, C.J.; Hasnip, P.J.; Clark, S.J.; Payne, M.C. First-principles simulation: Ideas, illustrations and the castep code. J. Phys. Condens. Matter 2002, 14, 2717-2744. [CrossRef]

27. Perdew, J.P.; Burke, K.; Ernzerhof, M. Generalized gradient approximation made simple. Phys. Rev. Lett. 1996, 77, 3865-3868. [CrossRef] [PubMed]

28. Hammer, B.; Hansen, L.B.; Nørskov, J.K. Improved adsorption energetics within density-functional theory using revised perdew-burke-ernzerhof functionals. Phys. Rev. B 1999, 59, 7413-7421. [CrossRef]

29. Perdew, J.P.; Zunger, A. Self-interaction correction to density-functional approximations for many-electron systems. Phys. Rev. B 1981, 23, 5048-5079. [CrossRef]

30. Ceperley, D.M.; Alder, B.J. Ground state of the electron gas by a stochastic method. Phys. Rev. Lett. 1980, 45, 566-569. [CrossRef]

31. Vanderbilt, D. Soft self-consistent pseudopotentials in a generalized eigenvalue formalism. Phys. Rev. B 1990, 41, 7892-7895. [CrossRef]

32. Boukhvalov, D.W.; Son, Y.W. Oxygen reduction reactions on pure and nitrogen-doped graphene: A first-principles modeling. Nanoscale 2012, 4, 417-420. [CrossRef] [PubMed]

33. Monkhorst, H.J.; Pack, J.D. Special points for brillouin-zone integrations. Phys. Rev. B 1976, 13, 5188-5192. [CrossRef]

34. Koelling, D.D.; Harmon, B.N. A technique for relativistic spin-polarised calculations. J. Phys. C Solid State Phys. 1977, 10, 3107-3114. [CrossRef]

35. Kresse, G.; Furthmüller, J. Efficiency of ab-initio total energy calculations for metals and semiconductors using a plane-wave basis set. Comput. Mater. Sci. 1996, 6, 15-50. [CrossRef]

36. Chen, W.H.; Yu, C.F.; Cheng, H.C.; Lu, S.T. Crystal size and direction dependence of the elastic properties of $\mathrm{Cu}_{3} \mathrm{Sn}$ through molecular dynamics simulation and nanoindentation testing. Microelectron. Reliab. 2012, 52, 1699-1710. [CrossRef]

37. Li, S.; Ouyang, C. First principles study of wurtzite and zinc blende GaN: A comparison of the electronic and optical properties. Phys. Lett. A 2005, 336, 145-151. [CrossRef]

38. Qin, H.B.; Luan, X.H.; Feng, C.; Yang, D.G.; Zhang, G.Q. Mechanical, thermodynamic and electronic properties of wurtzite and zinc-blende GaN crystals. Materials 2017, 10, 1419. [CrossRef] [PubMed] 
39. Zoroddu, A.; Bernardini, F.; Ruggerone, P.; Fiorentini, V. First-principles prediction of structure, energetics, formation enthalpy, elastic constants, polarization, and piezoelectric constants of $\mathrm{AlN}$, GaN, and $\mathrm{InN}$ : Comparison of local and gradient-corrected density-functional theory. Phys. Rev. B 2000, 64, 314-319. [CrossRef]

40. Stampfl, C.; Walle, C.G.V.D. Density-functional calculations for III-V nitrides using the local-density approximation and the generalized gradient approximation. Phys. Rev. B Condens. Matter. 1999, 59, 5521-5535. [CrossRef]

41. Bernardini, F.; Fiorentini, V.; Vanderbilt, D. Accurate calculation of polarization-related quantities in semiconductors. Phys. Rev. B 2001, 63, 193201. [CrossRef]

42. Detchprohm, T.; Hiramatsu, K.; Itoh, K.; Akasaki, I. Relaxation process of the thermal strain in the $\mathrm{GaN} / \alpha-\mathrm{Al}_{2} \mathrm{O}_{3}$ heterostructure and determination of the intrinsic lattice constants of GaNfree from the strain. Jpn. J. Appl. Phys. 1992, 31, L1454-L1456. [CrossRef]

43. Kanoun, M.B.; Goumri-Said, S.; Merad, A.E.; Merad, G.; Cibert, J.; Aourag, H. Zinc-blende AiN and GaN under pressure: Structural, electronic, elastic and piezoelectric properties. Semicond. Sci. Technol. 2004, 19, 1220. [CrossRef]

44. Nye, J.F. Physical Properties of Crystals: Their Representation by Tensors and Matrices; Oxford University Press: Oxford, UK, 1985.

45. Hill, R. The elastic behaviour of a crystalline aggregate. Proc. Phys. Soc. Sect. A 1952, 65, 349-354. [CrossRef]

46. Kim, K.; Lambrecht, W.R.; Segall, B. Elastic constants and related properties of tetrahedrally bonded BN, AlN, GaN, and InN. Phys. Rev. B 1996, 53, 16310. [CrossRef]

47. Polian, A.; Grimsditch, M.; Grzegory, I. Elastic constants of gallium nitride. J. Appl. Phys. 1996, 79, 3343-3344. [CrossRef]

48. Shimada, K.; Sota, T.; Suzuki, K. First-principles study on electronic and elastic properties of BN, AlN, and GaN. J. Appl. Phys. 1998, 84, 4951-4958. [CrossRef]

49. Wright, A.F. Elastic properties of zinc-blende and wurtzite AlN, GaN, and InN. J. Appl. Phys. 1997, 82, 2833-2839. [CrossRef]

50. Fan, Q.; Chai, C.; Wei, Q.; Yang, J.; Zhou, P.; Zhang, D.; Yang, Y. A new phase of GaN. J. Chem. 2016, 2016, 8612892. [CrossRef]

51. Chen, X.; Liang, J.; Xu, Y.; Xu, T.; Jiang, P.; Yu, Y.; Lu, K. Structure and debye temperature of wurtzite GaN. Mod. Phys. Lett. B 1999, 13, 285-290. [CrossRef]

52. Xia, H.; Xia, Q.; Ruoff, A.L. High-pressure structure of gallium nitride: Wurtzite-to-rocksalt phase transition. Phys. Rev. B 1993, 47, 12925. [CrossRef]

53. Yonenaga, I. Hardness, Yield strength, and dislocation velocity in elemental and compound semiconductors. Mater. Trans. 2005, 46, 1979-1985. [CrossRef]

54. Nowak, R.; Pessa, M.; Suganuma, M.; Leszczynski, M.; Grzegory, I.; Porowski, S.; Yoshida, F. Elastic and plastic properties of GaN determined by nano-indentation of bulk crystal. Appl. Phys. Lett. 1999, 75, 2070-2072. [CrossRef]

55. Kisielowski, C.; Krüger, J.; Ruvimov, S.; Suski, T.; Ager, J., III; Jones, E.; Liliental-Weber, Z.; Rubin, M.; Weber, E.; Bremser, M. Strain-related phenomena in GaN thin films. Phys. Rev. B 1996, 54, 17745. [CrossRef]

56. Luo, B.; Wu, X.; Li, G. Electronic structure, elastic and thermal properties of semiconductor GaX (X $=\mathrm{N}, \mathrm{P}$, As, $\mathrm{Sb}$ ) with zinc blende from first-principles calculation. Int. J. Mod. Phys. B 2014, 28, 1450183. [CrossRef]

57. Fan, Q.; Chai, C.; Wei, Q.; Yang, Y. Thermodynamic, elastic, elastic anisotropy and minimum thermal conductivity of $\beta-\mathrm{GaN}$ under high temperature. Chin. J. Phys. 2017, 55, 400-411. [CrossRef]

58. Vurgaftman, I.; Meyer, J.R.; Ram-Mohan, L.R. Band parameters for III-V compound semiconductors and their alloys. J. Appl. Phys. 2001, 89, 5815-5875. [CrossRef]

59. Bannikov, V.V.; Shein, I.R.; Ivanovskii, A.L. Electronic structure, chemical bonding and elastic properties of the first thorium-containing nitride perovskite TaThN 3 . Phys. Status Solidi RRL 2007, 1, 89-91. [CrossRef]

60. Pugh, S. XCII. Relations between the elastic moduli and the plastic properties of polycrystalline pure metals. Lond. Edinb. Dublin Philos. Mag. J. Sci. 1954, 45, 823-843. [CrossRef]

61. Pettifor, D.G. Theoretical predictions of structure and related properties of intermetallics. Mater. Sci. Technol. 1992, 8, 345-349. [CrossRef]

62. Lau, K.; Mccurdy, A.K. Elastic anisotropy factors for orthorhombic, tetragonal, and hexagonal crystals. Phys. Rev. B 1998, 58, 8980. [CrossRef] 
63. Panda, K.B.; Ravi Chandran, K.S. Determination of elastic constants of titanium diboride ( $\left.\mathrm{TiB}_{2}\right)$ from first principles using flapw implementation of the density functional theory. Comput. Mater. Sci. 2006, 35, 134-150. [CrossRef]

64. Zhang, J.M.; Zhang, Y.; Xu, K.W.; Ji, V. Anisotropic elasticity in hexagonal crystals. Thin Solid Films 2007, 515, 7020-7024. [CrossRef]

65. Zhang, J.M.; Zhang, Y.; Xu, K.W.; Ji, V. Young's modulus surface and poisson's ratio curve for cubic metals. J. Phys. Chem. Solids 2007, 68, 503-510. [CrossRef]

66. Rubio, A.; Corkill, J.L.; Cohen, M.L.; Shirley, E.L.; Louie, S.G. Quasiparticle band structure of AlN and GaN. Phys. Rev. B Condens. Matter 1993, 48, 11810. [CrossRef] [PubMed]

67. Heyd, J.; Scuseria, G.E.; Ernzerhof, M. Erratum:"Hybrid functionals based on a screened coulomb potential”. J. Chem. Phys. 2006, 124, 219906. [CrossRef]

68. Wei, S.H.; Zunger, A. Predicted band-gap pressure coefficients of all diamond and zinc-blende semiconductors: Chemical trends. Phys. Rev. B 1999, 60, 5404-5411. [CrossRef]

(C) 2018 by the authors. Licensee MDPI, Basel, Switzerland. This article is an open access article distributed under the terms and conditions of the Creative Commons Attribution (CC BY) license (http:/ / creativecommons.org/licenses/by/4.0/). 